\title{
Philonsorbonne
}

$14 \mid 2020$

Année 2019-2020

\section{Doctorales 2019}

Résumés des Doctorales de Philosophie (2019)

\section{OpenEdition}

\section{Journals}

Édition électronique

URL : https://journals.openedition.org/philonsorbonne/1491

DOI : 10.4000/philonsorbonne.149

ISSN : 2270-7336

Éditeur

Publications de la Sorbonne

Édition imprimée

Date de publication : 14 février 2020

Pagination : 119-134

ISSN : 1255-183X

\section{Référence électronique}

«Doctorales 2019 », Philonsorbonne [En ligne], 14 | 2020, mis en ligne le 28 février 2020, consulté le 03 mars 2022. URL : http://journals.openedition.org/philonsorbonne/1491 ; DOI : https://doi.org/

10.4000/philonsorbonne.1491

(c) Tous droits réservés 


\title{
Doctorales 2019 *
}

\author{
PHILOSOPHIE POLITIQUE
}

La dette publique menace-t-elle les institutions démocratiques?

Pierre DE SAINT-PHALLE

Qu'est-ce qu'une éducation démocratique?

Baptiste CORNARDEAU

\section{PHILOSOPHIE ANALYTIQUE}

Logique et normes dans la résolution des conflits

Sidiki Dosso

Peut-on prouver l'obligation? Réflexions sur les notions de preuves et de signification en logique déontique

Perceval PILLON

La philosophie de l'histoire et la morale de l'évolution

Nicolas PASTOR

La transposition des hétérochronies biologiques en psychologie

Sylvain BOSSELET

\section{PHILOSOPHER AUX XVII ${ }^{\mathrm{e}}$ ET XVIII ${ }^{\mathrm{e}}$ SIÈCLES}

La raison cartésienne est-elle structurée ?

Louis ROUQUAYROL

Rousseau, le dernier bon sauvage, ou l'âme de la République

Abir TAHA-AUDI

\section{PHILOSOPHIE CONTEMPORAINE}

La rythmique cosmologique du réel

au cour de la corrélation phénoménologique

Manfredi MORENO

Wittgenstein, essai d'unicité

Joseph SANKI KONGOLO

\footnotetext{
${ }^{(*)}$. Dans cette rubrique sont publiés les résumés des interventions ayant eu lieu lors des Doctorales de Philosophie organisées à la Sorbonne les 22, 23, 24 et 25 mai 2019, par les doctorants Ulysse Chaintreuil (PhiCo), Guillaume LAMBEY (PhiCo), Marie MichON (IHPST), Anca MiHalache (HIPHIMO) et Mélanie ZapPUlla (HIPHIMO).
} 


\section{ANTIQUITÉ ET MOYEN-ÂGE}

Peut-on définir sans la matière?

Aristote et les structures matérielles de la définition de la substance

Ulysse CHAINTREUIL

Je pense, je parle :

l'enjeu anthropologique de l'intellect chez Abélard

Angela Beatriz AvALOS

Tendere ad aeterna :

la voie temporelle vers l'éternité selon saint Augustin

Axel FouQueT

PANEL SUR LE « PRÉ-JUGÉ »

Peut-on dire que les émotions sont des jugements?

Marie MiCHON

La conception de la temporalité chez Hume et Rousseau

Iris DOUZANT

Le voyageur et son reflet :

le premier Heidegger, lecteur du De Veritate

Mathieu EYCHENIÉ

Le corps parachevé de sciences :

physique du «désir de savoir » chez Albert le Grand

Clémence MASSON

\section{ESTHÉTIQUE}

Philosophie et littérature : le moment des années 1960-1970

Anca MiHALACHE

Quel est l'objet de la perception misicale?

Quentin GAILHAC

\section{SPINOZA}

L'idée de décision chez le jeune Spinoza : le prologue du Traité de la réforme de l'entendement et la critique de la volonté Anne TEXIER

Les tension du concept d'ingenium dans la philosophie de Spinoza

Mélanie ZAPULLA

L'ordre social provient-il de passions antisociales?

Adam Ferguson et la selfish hypothesis

Sonia BOUSSANGE-ANDREI

Des choses mortes : Gilbert Simondon, critique de Spinoza 


\section{Philosophie PolitiQue}

\section{Pierre DE SAINT-Phalle - La dette publique menace-t-elle les institutions politiques?}

S'inscrivant dans le champ de la philosophie économique, cette communication est une contribution à l'analyse de la dimension normative du système d'endettement en tant qu'institution à part entière des États modernes. L'endettement de la sphère publique et les débats associés sont un objet d'étude précieux pour l'analyse de l'évolution des normes et de l'interprétation des principes fondamentaux qu'endossent les institutions publiques. David Hume (1748) et James Steuart (1767) eurent un débat sur l'influence du «crédit public» sur les institutions politiques. En échos, un débat contemporain existe entre Thomas Piketty (2012) et David Stasavage (2016) quant à l'enjeu social et démocratique de la dette publique. Je proposerai une synthèse de ces deux débats qui permettent de lier à la fois les enjeux institutionnels, financiers et politiques de la dette publique.

\section{Baptiste CORNARDEAU - Qu'est-ce qu'une éducation démocratique?}

John Dewey (1859-1952) a été un penseur de l'éducation et de la politique. Dans quelle mesure ces deux dimensions se rejoignent-elles? Quelles conséquences les formes éducatives propres à une société peuventelles avoir sur sa vie politique ? Et réciproquement, comment les modes de régulation politique de la vie sociale peuvent-ils se transcrire dans le domaine éducatif? Si la philosophie est bien, ainsi que le veut Dewey, une "théorie générale de l'éducation», il s'agira d'examiner en quoi elle se trouve convoquée à repenser ces problèmes et, ce faisant, à se reconstruire elle-même. 


\section{PhiLOSOPHIE ANALYTIQUe}

\section{Sidiki Dosso - Logique et normes dans la résolution des conflits}

Le thème de notre travail porte sur les relations entre la logique et les normes dans le processus de "prévention » voire de "résolution» des conflits internationaux. Cette partie de notre réflexion s'attelle à analyser certaines procédures de "bonne résolution» concernant les conflits internationaux. Partant des théories dites réalistes comme celle de Thomas Schelling concernant la décision interdépendante, à la théorie des jeux de Steven J. Brams, Hervé Moulin et Herbert Alexander Simon, nous essayerons de mettre en exergue la rationalité des acteurs politiques en particulier et des États en général face à la conflictualité. Certes, l'intérêt des États semble parfois primer sur celui des individus mais force est de constater que des négociations peuvent conduire à une politique de prévention des conflits. Il convient dès lors d'intégrer dans la «raison d'État» le principe « humain » qui ordonne de promouvoir et de protéger la vie en général - fût-elle celle de notre ennemi. Avec l'avènement du nucléaire, il semble impératif pour les décideurs politiques de passer d'une irrationalité pure à une forme parfaite de rationalité.

\section{Perceval Pillon - Peut-on prouver l'obligation? Réflexions sur les notions de preuves et de signification en logique déontique}

La logique déontique, en ce qu'elle s'attache aux notions d'obligation, de permission et d'interdiction, notions qui s'éloignent du champ classique de la logique mathématique, permet d'interroger le domaine et la possibilité de formalisation, tant d'un point de vue philosophique que du point de vue de la technicité des systèmes. Questionner la possibilité d'une logique de l'obligation et de ce que ce terme implique interroge la définition même de ce qu'est une logique et, de fait, ce qu'elle peut saisir de la notion ici en jeu.

Nous défendons l'importance du critère syntaxique dans la détermination de la logicité d'un système formel et de sa capacité expressive, notamment dans le cas d'une formalisation de concepts non mathématiques. Avoir une logique déontique et donc un ensemble d'opérateurs pour les raisonnements normatifs impliquerait donc qu'il est possible d'appliquer un traitement inférentialiste à ceux-ci. Il nous semble en effet crucial d'indiquer que le traitement formel d'une notion - ici l'obligation - ne doit pas se départir d'un traitement preuve-théorique, garant du caractère combinatoire du langage utilisé. Notre communication fournira ainsi une analyse de certains formalismes paradigmatiques (notamment les approches modales) pour la logique déontique sous l'angle défini. Cela 
nous permettra d'aborder une question inhérente à l'idée d'une logique qui s'attache aux expressions normatives: est-il possible de donner un traitement strictement syntaxique de la notion d'obligation et quel serait le résultat d'une telle démarche?

\section{Nicolas PASTOR - La philosophie de l'histoire et la morale de l'évolution}

La philosophie de l'histoire est un genre délaissé, abîmé par la faillite de ses plus illustres représentants. Empruntant la formule d'un Loisy, nous pourrions dire que Hegel annonçait l'absolu, et que c'est le postmodernisme qui est venu. Quant au marxisme, on a tôt fait de l'enterrer avec les régimes qui s'en réclamaient, pour proclamer avec Fukuyama (1989) la fin de l'histoire. Avec pessimisme, on pourrait s'en tenir au diagnostic d'Adorno (1966, p. 3): le monde était gros d'un changement «et il a avorté». Pourtant, la philosophie de l'histoire ne saurait périr de ses échecs. Les préoccupations environnementales, l'inquiétude portant sur l'avenir de notre espèce, les progrès des sciences qui nous donnent à contempler la majesté des temps passés et nous laissent penser ceux à venir, constituent un sol fertile sur lequel peut se développer une philosophie de l'histoire revivifiée. Parmi ces sciences, la théorie de l'évolution est riche de débats portant sur la nature du devenir, la finalité, la morale et le progrès. Tout autant de thèmes mobilisés par Kant (1784) dont la philosophie de l'histoire exigeait que l'on postule un plan caché de la nature; un plan investi d'une signification morale explicite et dominé par l'idée du progrès. Dans cette communication, nous interrogerons la possibilité d'une philosophie de l'histoire fondée sur notre connaissance de l'évolution. Celle-ci devra nécessairement se confronter aux thèmes que nous venons d'évoquer, considérant avec Simpson qu'ils sont étroitement connectés. Ce dernier pensait que la plupart des tentatives visant à tirer un enseignement et peut-être de fonder une morale de l'évolution "demandent que l'on essaie de découvrir ce que projetait l'évolution, ou même ce qu'elle semble vouloir, et qu'elles partent du principe que ce qui facilite ce dessein est moralement bien ». Voilà les perspectives que nous développerons.

\section{Sylvain BosseleT - La transposition des hétérochronies biologiques en psychologie}

Suite à la loi censément universelle de la récapitulation ontophylogénétique postulée par Haeckel vers la fin du XIX ${ }^{\mathrm{e}}$ siècle (le développement d'un individu récapitulerait celui de ses espèces ancestrales), Freud et Piaget supposent parfois que le développement d'un enfant « reparcourt» les âges mentaux «primitifs » de nos ancêtres. Au contraire, suite à la supposée néoténie humaine postulée par Bolk au début du XX siècle (les hommes actuels s'en tiendraient au stade juvénile de nos ancêtres), Lacan, Lorenz, Winnicott, Huizinga, Sloterdijk et d'autres 
supposent que notre développement mental est retardé. Par exemple, nous resterions joueurs plus longtemps que nos ancêtres.

Mais la biologie actuelle a abandonné (le caractère universel et holistique de) ces deux théories. Elle défend l'idée d'hétérochronie (changement de vitesse et de durée du développement du descendant par rapport à ses ancêtres), qui concerne plus précisément des organes et des parties d'organes de l'individu (au lieu d'un individu pris globalement). Nous tâchons de transposer cette théorie plus fine sur le plan psychologique, en nous demandant quelles capacités mentales actuelles seraient «sur-» ou «sous-développées » par rapport à nos ancêtres préhistoriques, et pour quelles raisons adaptatives.

Pour relier les développements psychologiques de nos ancêtres et de nos contemporains, nous convoquons d'un côté l'archéologie cognitive et de l'autre la psychologie du développement, notamment pour tenter de découper des stades de développement selon de nombreuses capacités mentales. Nous en dégageons la notion de "personnalité hétérochronique », qui permettrait en droit de définir quantitativement, par des tests, les différents niveaux d'aptitudes mentales atteints par chaque individu selon les différents types d'intelligences reconnues.

Cet outil permettrait d'interpréter philosophiquement bien des aspects de l'histoire humaine, avec pour perspective de compléter la notion de nature humaine d'un point de vue naturaliste. La grande flexibilité développementale de nombreuses capacités psychologiques différenciées constituerait une caractéristique plus que jamais vitale de l'humanité.

\section{PHILOSOPHER AUX XVII ${ }^{\mathrm{e}}$ ET XVIII ${ }^{\mathrm{e}}$ SIÈCLES}

\section{LOUIS ROUQUAYROL - La raison cartésienne est-elle structurée ?}

On s'accorde parfois à penser (depuis J. Laporte notamment) que la raison, chez Descartes, parce qu'elle serait une simple capacité de bien juger, n'est en aucune façon structurée. Deux éléments au moins amènent à douter qu'il en soit ainsi: (1) l'âme a été formée par Dieu avec des idées innées dont on comprendrait mal qu'elles ne fussent pas articulées entre elles, au moins de façon élémentaire ; (2) certaines «lois» ont été imprimées par Dieu en notre âme (selon les termes de la lettre à Mersenne du 15 avril 1630) qui se rapportent à celles qui, instituées dans la nature, structurent actuellement le monde.

Par conséquent, la puissance de bien juger n'est pas le tout de la raison : la lumière naturelle, pourvoyeuse d'axiomes et de notions communes qui relient entre elles des notions simples, est déterminante dans le processus 
de connaissance. De cette fonction de liaison, on tire deux singularités de la pensée cartésienne qui restent à examiner (et que l'on étudiera en particulier à partir de la notion commune " pour penser, il faut être »). (1) L'opposition entre Descartes et Leibniz (notamment avec Belaval) ayant renforcé l'idée d'une raison cartésienne sans structure, il s'agit de montrer que la raison, chez Descartes, enveloppe une structuration partielle de notre processus de connaissance - et non, comme chez Leibniz, la structuration parfaite qui serait celle d'un "automate spirituel ». (2) Par ailleurs, cette structuration doit être comprise dans le cadre de la thèse de la création des vérités éternelles, impliquant, pour celle-ci, une certaine contingence (certes incompréhensible).

\section{Abir TAHA-AUd - Rousseau, le dernier bon sauvage, ou l'âme de la République}

Tout comme le Mahatma Gandhi plusieurs siècles plus tard affirmera : " ma vie est mon message », de même, la vie et la pensée de Jean-Jacques Rousseau sont inextricablement liées. On ne peut séparer Rousseau le rêveur, promeneur solitaire et romantique, du Rousseau père de la Révolution Française et de ses idéaux républicains de liberté, d'égalité et de fraternité. Malheureusement, l'idéalisme innocent, ou l'innocence idéaliste de Rousseau sont une denrée rare dans notre monde moderne où règnent la cupidité, la compétitivité maladive, le mercantilisme, l'égoïsme mesquin, la guerre de tous contre tous dans sa version libérale lockéenne plutôt que hobbesienne. Disons-le sans ambiguïté : nous sommes encore loin de l'idéal républicain d'une communauté de citoyens libres et égaux où règne l'état de droit, c'est-à-dire l'état moral prônant la justice universelle. En effet, liberté et justice ne vont malheureusement pas toujours de pair; or sans justice, peut-il y avoir de véritable égalité ? Et sans égalité, peut-il y avoir de véritable liberté ? D'où l'importance d'un retour à l'esprit de Rousseau et d'une renaissance de cet esprit afin de passer du libéralisme au républicanisme, de l'État qui protège les riches à l'État de tous les citoyens, l'Etat de Droit, seule véritable méritocratie.

Nous nous proposons donc dans ce travail de démontrer l'actualité de la pensée de Rousseau et d'analyser la crise de la modernité à travers une critique rousseauiste du libéralisme. Nous démontrerons ensuite que la philosophie politique de Rousseau - et notamment son idéal républicain permet de concilier citoyenneté et marché libre, liberté et égalité, patriotisme et humanisme, ouvrant ainsi une troisième voie entre libéralisme et socialisme: le républicanisme, qui va au-delà des clivages politiques et de l'opposition entre droite et gauche, pour constituer une théorie pour tous les citoyens. 


\section{PHILOSOPHIE CONTEMPORAINE}

\section{Manfredi Moreno - La rythmique cosmologique du réel au caur de la corrélation phénoménologique}

Le sensible sollicite une dimension universelle prise en charge par l'a priori universel de corrélation et le phénomène de la phénoménologie révèle cette dimension en termes d'une certaine réalisation d'un monde. Ainsi, le sensible s'achemine vers l'universel car il accueille le monde en son sein, de sorte que le sujet et le monde sont bien inséparables, nécessité eidétique de la corrélation, sauf qu'ils ne se rencontrent jamais, nécessité ontologique de la corrélation.

\section{Joseph SANKI Kongolo - Wittgenstein, essai d'unicité}

Cet exposé vise à présenter une lecture philosophique qui met en valeur l'idée selon laquelle la pensée de Wittgenstein, contrairement à ce que son apparence nous a déjà fait croire, peut être vue et lue comme étant pensée unique et cohérente, une pensée qui s'inscrit dans une logique de continuité et de cohérence possible. Il s'agit d'un essai polémique, dans un contexte où l'idée d'un troisième Wittgenstein est déjà soutenue. L'une des prises de positions les plus imposantes est celle de D. Moyal-Sharrock qui préface De la certitude pour le consacrer en même temps comme étant le chef d'œuvre du troisième Wittgenstein. Loin de remettre en question la légitimité philosophique d'une telle hypothèse, nous souhaitons simplement proposer ce que nous considérons comme étant un nouveau regard ou du moins un autre regard possible à propos de Wittgenstein, à savoir celui qui trouve racine dans l'idée de l'unité et de la continuité logique d'une pensée qui s'est entièrement structurée autour de la question de la vérité. Il est donc clair que nous nous inscrivons ici dans la logique du nouveau Wittgenstein mais en y apportant en renfort quelques arguments que nous exposerons à l'occasion de ces Doctorales.

\section{ANTIQUité ET MOYEN-ÂGE}

\section{Ulysse Chaintreuil - Peut-on définir sans la matière? Aristote et les structures matérielles de la définition de la substance}

Nous nous proposons d'éclairer le rôle des structures matérielles qui composent la définition, en s'intéressant plus particulièrement à la place 
que prend l'énoncé des parties matérielles dans l'énoncé des définitions des substances, en particulier des substances sensibles. Par exemple, la définition de l'âme, donnée au début du livre 2 du De anima, semble devoir impliquer, en un certain sens, l'énoncé du corps ( « l'âme est un corps possédant la vie en puissance »). À travers une analyse des chapitres 10 et 11 du livre $Z$ de la Métaphysique, nous essaierons de montrer que ce qui est utilisé dans la définition comme structure matérielle de la forme, ce n'est pas la matière comme matière, mais plutôt la matière comme déjà issue d'une composition hylémorphique, constituée d'un certain nombre de déterminations formelles. Ce qui joue le rôle de matière dans l'énoncé définitionnel, ce sont donc les niveaux de formalisation au-dessous du niveau de la substance composée, laissant donc, dans la définition, une place à la matière prise dans un certain sens, qu'il s'agit de préciser.

\section{Angela Beatriz Avalos - Je pense, je parle: l'enjeu anthropologique de l'intellect chez Abélard}

Ce que nous nommons «psychologie » abélardienne est davantage une " noétique »: elle ne porte pas, en vérité, sur la psyché, sur l'âme, mais sur l'intellect et l'acte de la pensée. Et la portée de cette doctrine, mêlée à une théorie du langage (à une "philosophie dans le langage ", dans les mots de Jean Jolivet), est anthropologique, dès lors que toute pensée veut être « dite » et qu'il n'y a que l'homme qui parle. Comment, donc, pensée et parole adviennent-elles ? C'est la question qu'il faut poser, sur la base d'un lexique flou, instable, pré-scolastique si l'on veut, afin d'établir comment dans le monde latin de Pierre Abélard - un monde sans le De anima d'Aristote s'esquisse une forme, à la fois précise et confuse, d'anthropologie noétique.

\section{Axel Fouguet - Tendere ad aeterna : la voie temporelle vers l'éternité selon saint Augustin}

La mutabilité temporelle de notre nature creuse en nous la distance avec celui dont nous sommes l'image, le Dieu éternel. La condition temporelle actuelle n'est pas, pour Augustin, la vérité de l'existence humaine, mais est appelée à être dépassée par une participation à l'éternité divine. Cependant, cet état d'union à l'éternité divine est eschatologique, et l'homme ne peut le revendiquer de son vivant, de sorte que sa temporalité est irréductible. Telle est la tension traversant la condition humaine terrestre : destinée à l'éternité, elle ne peut pour autant échapper à sa temporalité. Cette tension interdit l'illusion d'une union directe avec l'éternel par nos propres forces. Mais elle ouvre aussi une nouvelle voie, celle qui consiste, avec l'aide de la grâce, à «infuser» autant que possible l'éternité dans notre temporalité et à adopter, dans le temps même, un nouveau rapport au temps. C'est ce mode d'existence temporelle tourné temporellement vers l'éternel que nous nous proposons de présenter : comment restaurer un rapport authentique au temps qui, dès cette vie, le fasse déjà tendre vers l'éternité ? 


\section{PANEL SUR LE « PRÉ-JUGÉ »}

\section{Marie Michon - Peut-on dire que les émotions sont des jugements?}

Les choix méthodologiques d'élaboration d'une théorie contraignent les choix philosophiques, c'est pourquoi nous proposons une analyse de l'assimilation des émotions à des jugements (Solomon (1973; 2005), Nussbaum (2001)) à partir des lacunes méthodologiques pour mettre, ensuite, en lumière les problèmes conceptuels.

La causalité est un concept dangereux en philosophie des émotions parce qu'il sous-tend une idée de sens et de rationalité. Depuis l'antiquité et les Stoïciens, nous voyons des disputes de pouvoir entre le mental et le corps; l'hypothèse cognitiviste est une tentative de plus de démontrer la suprématie du mental, notamment après les thèses de James (1884). L'intentionnalité des émotions se veut volontaire; le contrôle accessible et responsabilisant. La philosophie des émotions-jugements entend être un coup de marteau final aux passions : c'est la psyché raisonnable qui s'adapte aux faits. Cependant, c'est justement dans l'expérience que l'on teste les limites. Deux thèses sous-tendent le courant: la thèse constitutive et la thèse causative, celle qui considère que je ressens lorsque je juge et celle qui considère que je ressens parce que je juge. Nous analyserons, en écho aux travaux de Scarantino (2010), ces deux thèses à travers les propriétés qu'elles attribuent et aux émotions, et aux jugements. En effet, l'argumentation repose sur une tentative de lecture des propriétés d'un objet à la lumière de celles de l'objet auquel on veut l'assimiler ; or les définitions de chacun des concepts en jeu sont multiples et ne doivent pas être choisies à la légère - il en va ainsi des propriétés correspondantes. Plutôt que de dire que les émotions ne sont pas des jugements, nous montrerons qu'elles ne peuvent, en réalité, pas en être.

\section{Iris Douzant - La conception de la temporalité chez Hume et Rousseau}

Les décisions méthodologiques qui sont à l'œuvre dans le Traité de la nature humaine et dans Émile ou de l'éducation mettent en évidence l'attention que Hume et Rousseau accordent à l'expérience : c'est bien l'observation des comportements humains qui conditionne aussi bien la science de la nature humaine humienne que la théorie de l'homme rousseauiste. Pour les deux philosophes, la nature humaine se révèle au sein de la variété des circonstances : il s'agit de réunir les observations et de les comparer. Chacun entend bien mettre au jour une constance voire une invariance qui serait dissimulée par les variations, en partant 
de ces variations et en les analysant. Néanmoins, et notamment parce que l'entreprise de Hume consiste à introduire la méthode expérimentale newtonienne dans les sujets moraux, celui-ci fait un usage scientifique du temps : ce que l'histoire des hommes révèle, ce sont des fréquences. Ce ne sont pas des époques qui découpent le temps humain, qui est conçu de façon uniforme. La variété du temps est, pour Hume, un foisonnement que la science de la nature humaine démantèle : l'uniformité est toujours la cause de la variété. Pour Rousseau, au contraire, la variété qui se donne à voir dans la société n'est pas tant un foisonnement à partir duquel on pourrait inférer les principes de la nature humaine qu'un recouvrement voire une corruption de cette nature. Puisque pour Rousseau, la vie en société recouvre la nature humaine d'une condition sociale, la généralité ne peut pas être simplement inférée de la réunion des observations et de leurs comparaisons. Il faut, en outre, retrancher des observations ce qui n'est proprement qu'artificiel, c'est-à-dire ce qui est causé par l'histoire de l'homme. Ainsi, l'analyse de la prise en compte par Rousseau de l'historicité des comportements humains, confrontée à celle de la conception scientifique qu'a Hume du temps humain, nous permettra-t-elle de mettre en lumière deux conceptions très différentes des rapports entre la nature et de la société.

\section{Mathieu EYCHENIÉ - Le voyageur et son reflet: le premier Heidegger, lecteur du De Veritate}

Dès sa thèse d'habilitation, Heidegger présente Thomas d'Aquin comme un partisan de l'Abbildtheorie. La connaissance, selon Thomas, consisterait en une image (au sens de copie, reproduction, ou encore reflet) de la chose, de sorte que l'âme, après la chute - dans les termes de la scolastique médiévale: l'homo viator, le voyageur -, ne percevrait qu'indirectement les étants extra-mentaux. Mieux, le caractère spéculaire de la connaissance se verrait, en deux sens, redoublé, le voyageur thomasien se réfléchissant lui-même dans son acte de connaissance, et décelant dans la chose connue un reflet de la science divine.

L'enjeu de notre travail est donc d'une part d'exposer en détail la lecture heideggérienne de Thomas, en montrant comment elle évolue au gré des propres conceptions du philosophe de Messkirch : opposée, dans la thèse d'habilitation, à la théorie scotiste de la signification (qui préfigure à la fois la philosophie néokantienne des valeurs et la thématisation husserlienne de l'intentionnalité), la théorie thomasienne de la connaissance est présentée, dans le cours de 1923-1924, l'Introduction à la recherche phénoménologique, comme une étape décisive dans l'histoire du recouvrement de l'essence anté-prédicative ou anté-judicative de la vérité. Il s'agit, d'autre part, de faire apparaître le caractère réducteur de cette seconde interprétation. L'auteur d'Être et temps - qui déclare, dans son cours de 1927, Les Problèmes fondamentaux de la phénoménologie, que « la scolastique ignore la doctrine de l'intentionnalité » - fait peu de cas du concept thomasien d'intention, voire le méconnaît partiellement: d'un 
côté, il ne marque pas suffisamment la différence entre l'être réel et l'être intentionnel, de l'autre, il ne tient pas compte du dialogue engagé par Thomas avec les théories arabes de la perspective.

\section{Clémence MASSON - Le corps parachevé de sciences : physique du «désir de savoir » chez Albert le Grand}

Lorsqu'il commente les premières lignes de la Métaphysique, d'après lesquelles «tous les hommes par nature désirent savoir », Albert le Grand digresse : il prend de la distance avec la lettre aristotélicienne pour répondre à un certain nombre de problèmes, le principal étant que, tandis que « tous les hommes par nature désirent savoir », chaque homme n'est pas savant, ni même n'aspire à l'être.

À ce problème, un classique du péripatétisme, de nombreuses réponses se sont tour à tour constituées: réponses politiques, religieuses, logiques. Celle d'Albert est à la fois éthique et naturaliste. Les sciences qu'il mobilise sont en effet la physique, l'éthique et la médecine ; il les mobilise pour dire que si chaque homme, parce qu'il possède un intellect, aspire naturellement à le parfaire, chacun, en vertu de ses mœurs, peut corrompre cette nature, ou, par sa complexion propre, ne pas pouvoir la réaliser au maximum.

C'est ainsi dire que la réalisation de l'intellectualité humaine repose, presque avant tout, sur le désir. Devenir un homme, chez Albert, se joue d'abord au niveau du désir, compris à la fois comme une aspiration originelle à épouser parfaitement sa nature et à la fois comme une faculté ornée d'habitus rendant capable de cette nature. Devenir un homme, c'est en premier lieu pouvoir, par son corps, et devoir, par ses habitudes, désirer et se maintenir dans un désir proprement humain. S'y maintenir jusqu'au bout: jusqu'à connaître les pensées divines. On s'est ici intéressé à la dimension physique et médicale du devenir intellectuel.

\section{ESTHÉTIQUE}

\section{Anca Mihalache - Philosophie et littérature : le moment des années 1960-1970}

À partir des années 1960-1970, on voit apparaitre des philosophies se démarquant par une attention manifeste de l'écriture pour le style et par un intérêt pour de nouveaux objets d'étude. Le rapprochement entre la philosophie et la littérature est une conséquence du renversement paradigmatique qui s'est opéré pendant ces années, consistant en un certain affranchissement à l'égard des modèles discursifs traditionnels. Ces années 
coïncident avec une ouverture de la philosophie vers la littérature, se manifestant soit par une recherche des sources épistémiques dans le discours littéraire - dans ce cas, la littérature est revendiquée comme expérience de pensée ou cristallisation des discours -, soit par une attention accrue de l'écriture philosophique pour le style. Ces philosophies supposent une certaine conceptualité de la littérature, qui s'inscrit dans des réseaux de sens mythiques, historiques, philosophiques, politiques.

\section{Quentin GAILHAC - Quel est l'objet de la perception musicale?}

Constituant dans les Leçons sur la conscience intime du temps de Husserl un exemple privilégié d'objet temporel, la mélodie n'en demeure pas moins un objet problématique pour la perception musicale. Objet dont la constitution se passe d'une désignation langagière univoque et dont la délimitation, d'apparence immédiate, révèle dans la perception une somme de déterminations historiques, la mélodie est, en effet, le nom d'un problème qui informe et oriente le programme d'une phénoménologie de la perception musicale. Les structures de la rétention et de la protention, en se révélant insuffisantes pour expliquer par elles-mêmes l'identification d'un objet pour la perception, dissimulent aussi les conditions sous lesquelles cet objet non conceptuel se constitue toutefois selon une norme. Notre capacité à retenir le tout juste passé et à anticiper ce qui va suivre, même de manière inadéquate, inscrit en réalité notre perception dans les horizons déterminés de l'expérience, et trouverait à s'expliciter, chez Husserl lui-même, dans ce que le paragraphe 47 des Ideen I avait désigné sous le nom d'une théorie des types; théorie au regard de laquelle Alfred Schütz pensait pouvoir prendre en charge l'historicité constitutive de la perception musicale et de son objet. Non que l'objet de la perception musicale soit en lui-même un type - car un type se situe au niveau des règles que possède la perception et grâce auxquelles un enchaînement de notes peut devenir un objet - : ce sont plutôt les règles à valeur de type qui font l'objet d'une appropriation subjective et qui définissent, pour la perception, la nature spécifique de son objet.

\section{SPINOZA}

\section{Anne Texier - L'idée de décision chez le jeune Spinoza: le prologue $d u$ Traité de la réforme de l'entendement et la critique de la volonté}

La critique de la volonté, qui trouve sa forme définitive dans les propositions 48 et 49 de la deuxième partie de l'Éthique, est déjà présente dans le Court traité (dont on pense qu'il est rédigé vers la fin de 1660). Spinoza qualifie la volonté de " fiction », $d$ ' " être de raison ». Cette thèse est reprise en 1661 dans la Lettre 2, ainsi que dans la préface des Principes de la 
philosophie de Descartes en 1663. La question que nous nous posons est de savoir si Spinoza, dans les années qui précèdent, est déjà en possession de cette thèse, ou si au contraire il y eut chez lui rupture ou évolution sur ce point. Un seul texte peut nous aider à répondre à cette question, le Traité de la réforme de l'entendement, dont on sait maintenant qu'il date vraisemblablement de 1656, et dont le prologue est le récit d'une décision. Faut-il pour autant suivre sur ce point certains commentateurs et voir dans le prologue une "théorie sous-jacente de la volonté » absente dans les œuvres suivantes? Nous faisons l'hypothèse inverse et tentons d'établir, à la lecture du texte, que si décision il y a, il ne s'agit en aucune façon d'une décision volontaire, mais d'un processus décisionnel entièrement rationnel. Que la future critique de la volonté et la réduction des volitions aux idées n'apparaisse pas explicitement dans le Traité de la réforme de l'entendement, cela est manifeste. Mais ce que montre le prologue, nous semble-t-il, c'est qu'en amont de cette déconstruction, Spinoza, dans ce récit des différentes étapes de sa résolution, se passe tout bonnement de la volonté. Il n'en a pas besoin.

\section{Mélanie ZAPPULLA - Les tensions du concept d'ingenium dans la philosophie de Spinoza}

Dans la philosophie de Spinoza, l'ingenium apparaît comme le lieu d'une tension entre imitation d'autrui et invention de soi. Le concept d'ingenium, dans l'œuvre de Spinoza, peut se traduire par «esprit», « tempérament», « complexion» ou encore « génie». D'un point de vue épistémique, l'ingenium peut référer à l'esprit de n'importe quel individu, et plus rarement à un esprit exceptionnellement doué. L'ingenium au sens éthique peut se définir comme un "complexe d'affects sédimentés constitutifs d'un individu, de son mode de vie, de ses jugements et de son comportement " (Chantal Jaquet, Les Transclasses ou la non-reproduction, Paris, PUF, 2014). Dans son acception politique, le concept d'ingenium renvoie à l'entité collective que constitue le peuple ou la «multitude» dans sa singularité distinctive.

Il s'agira d'évoquer lors de cette présentation des problèmes structurant les recherches effectuées dans le cadre de mon travail de thèse, et plus spécifiquement :

- un problème d'ordre éthique: si l'imitatio affectuum apparaît comme un principe structurant de l'ingenium, comment un individu peut-il n'être pas qu'un simple produit de l'imitation d'autrui, et accéder ainsi à l'invention de soi, entendue comme élaboration d'une complexion singulière ?

- un problème de nature politique: peut-on sauver l'unité du sujet politique en dépit des tensions qui structurent les rapports entre l'ingenium d'un peuple et les ingenia ou esprits des divers individus qui le composent? 


\section{Sonia BOUSSANGE-ANDREI - L'ordre social provient-il de passions antisociales? Adam Ferguson et la selfish hypothesis}

L'hypothèse égoïste [selfish hypothesis] soutient que toute action est motivée par l'amour de soi [self-love]. Les êtres humains ne seraient pas naturellement sociables et soucieux du bien de leurs semblables, mais poursuivraient sans relâche leurs intérêts privés, même lorsqu'ils semblent agir avec désintéressement. Cette hypothèse a été défendue de manière frappante par Bernard Mandeville (1670-1733) dans La Fable des abeilles, où il avance l'idée paradoxale selon laquelle "les vices privés font le bien public » (Private Vices, Publick Benefits) ${ }^{1}$. Autrement dit, bien qu'ils apparaissent, à première vue, comme délétères à l'ordre social, les motifs égoïstes contribuent au contraire à cet ordre, s'ils sont correctement manipulés. Les vices et passions antisociales comme la cupidité et l'orgueil se révèlent même cruciaux à la constitution d'un ordre social bien fondé. La thèse défendue par Mandeville a essuyé de nombreuses critiques, parmi lesquelles celle d'Adam Ferguson (1723-1816), professeur de philosophie morale et pneumatique ${ }^{2}$ à l'université d'Édimbourg. Dans le cadre de sa réflexion sur la société civile ${ }^{3}$, Ferguson repose à nouveaux frais la question de l'ordre social et examine cette hypothèse à laquelle il adresse plusieurs griefs. Il s'attache tout d'abord à argumenter en faveur de l'idée selon laquelle une connaissance morale est possible et qu'elle est motivante. Il montre également quelles sont les conséquences désastreuses de la recherche de l'intérêt privé en toute chose. Mais il n'affirme pas pour autant que la sociabilité soit uniquement fondée sur la bienveillance des êtres humains. En soutenant que le conflit est au cœur de la vie sociale, qu'il existe des raisons morales d'agir qui ne sont pas seulement bienveillantes, il souligne le rôle de passions désintéressées comme l'animosité et offre ainsi un paradigme explicatif original de l'ordre social, qui va à l'encontre de l'anthropologie égoïste.

\section{Nicolas LeMA HABASH - Des choses mortes : Gilbert Simondon, critique de Spinoza}

Spinoza n'occupe pas une place centrale dans le corpus de Simondon. Notre argument soutient que cette négligence de Simondon par rapport à l'œuvre de Spinoza peut s'expliquer par le fait que Simondon ne pouvait pas se concevoir comme le développeur d'une philosophie spinoziste. Pour Simondon, les conséquences de la conception spinoziste de conatus étaient inacceptables du point de vue de la genèse des organismes vivants qu'il a étudiée depuis sa thèse doctorale, L'Individuation à la lumière des notions de forme et d'information (publiée intégralement en 2005). La conséquence

1. Bernard Mandeville, La Fable des Abeilles, trad. L. et P. Carrive, Paris, Vrin, 2010, p. 21.

2. La philosophie de l'esprit était nommée ainsi au XVIII' siècle.

3. Cf. Adam Ferguson, An Essay on the History of Civil Society [1767], éd. F. Oz-Salzberger, Cambridge, Cambridge University Press, 1995. 
centrale de la notion de la notion de conatus spinoziste, d'après Simondon, est l'impossibilité de créer des organismes vivants dans la mesure où chez Spinoza, il n'y a aucune place pour une pensée de la "potentialité », une conception clé pour le développement de tout être vivant. Selon Simondon, "Vivre est perpétuer une permanente naissance relative» qui ne peut se produire qu'à travers le temps. Cette perpétuation des naissances, ce déphasage constant dans le temps est précisément ce que la philosophie de Spinoza ne peut pas concevoir : la substance est déjà éternelle, sans temporalité, et les modes sont des automates incapables de se déphaser. 